\title{
Refuerzo de Competencias interdisciplinares en las titulaciones de Arquitectura e Ingeniería de Edificación. Workshop docente "Estructuras singulares"
}

\author{
Igor Fernández-Plazaola ${ }^{a}$, Francisco Sanchis-Sampedro ${ }^{b}$, Jaime Llinares-Millán ${ }^{\mathrm{c}}$ y \\ Jose Miguel Molines Cano ${ }^{d}$ \\ ${ }^{a}$ Universitat Politècnica de València (UPV) Departamento de Organización de empresas \\ iplazaola@doe.upv.es, ${ }^{b}$ UPV Departamento de expresión gráfica arquitectónica fsanchis@ega.upv.es \\ ${ }^{c}$ UPV Departamento de construcciones arquitectónicas jaillimi@csa.upv.es ${ }^{\mathrm{d}}$ UPV Departamentos de \\ mecánica de los medios continuos y teoría de estructuras jomoca1@upv.es.
}

\begin{abstract}
Bachelor degrees in Architecture and Building Engineering develop several learning skills outcomes which are acquired in different subjects. These subjects rarely find a framework in which sharing the acquired knowledge and, therefore, to understand or experience the skills and competences as a whole.

In this workshop foldable singular surfaces are created, this competence is acquired after completing Descriptive Geometry, Structures I and Architectural Projects courses. At the workshop a unique foldable surface is explained, this can be created using tensioned and rigid elements. Students should understand the figure geometrically and solve it spatially using basic and recycled materials (broomsticks, screws and strings) and adjusting tensioned elements to eventually cover a $4 \times 4 \mathrm{~m}$ area.
\end{abstract}

The workshop development is divided into two different phases, in the first one different base modules are created (in groups of 3-4 people) once finished its folding operation is shown in the classroom. In the second phase the different modules connection is studied and different types of links between modules are checked.

After the workshop a survey was carried out in order to assess the whole experience from the theoretical content, the materials, the spaces and the involved competences.

Keywords: Singular surfaces, tensioned structures, generic skills, descriptive geometry, architectural surfaces

(cc) EY-NC-ND 2015, Universitat Politècnica de València

Congreso In-Red (2015) 
Refuerzo de Competencias interdisciplinares en las titulaciones de Arquitectura e Ingeniería de Edificación. Workshop docente "Estructuras singulares"

\begin{abstract}
Resumen
En las titulaciones de arquitectura y de Ingeniería de Edificación existen varias competencias cuyos resultados de aprendizaje se adquieren en diferentes asignaturas. Estas asignaturas, en pocas ocasiones encuentran un marco donde poner en común los conocimientos adquiridos y, por lo tanto, poder entender o experimentar la competencia en su totalidad
\end{abstract}

En este workshop se trabaja la creación de superficies singulares desplegables, competencia la cual se adquiere tras cursar las asignaturas Geometría descriptiva, Estructuras I y Proyectos arquitectónicos. En el taller se explica una superficie singular desplegable, la cual se puede crear empleando elementos tensionados y elementos rígidos. Los alumnos deben entender geométricamente la figura, resolverla espacialmente empleando materiales reciclados (palos de escoba, tornillería y lienzas) y ajustar los elementos tensionados para, finalmente cubrir un espacio de $4 \times 4 m$.

El desarrollo se realiza en dos partes, en una primera fase se crean diferentes módulos base (en grupos de 3-4 personas) y se muestra su funcionamiento en el aula. En la segunda fase se estudia la unión de los diferentes módulos y se comprueban los diferentes tipos de enlaces.

Tras el taller se han pasado encuestas a los alumnos para comprobar la experiencia desde el punto de vista de refuerzo o comprensión de la competencia.

Palabras clave: Superficies singulares, estructuras tensionadas, competencias interdisciplinares, geometría descriptiva, arquitectura de superficies

\title{
1. Introducción
}

La geometría desde siempre ha sido el instrumento que ha permitido a los arquitectos, de muy diversas formas, unir la creatividad del proceso de diseño, con las necesidades estructurales y constructivas. Es por tanto una herramienta fundamental de diseño y análisis de la Arquitectura y su uso en fases tempranas de proyecto es imprescindible debido a que soluciona problemas posteriores durante la construcción y el comportamiento estructural del edificio.

Dejando de lado criterios estructurales y constructivos, y centrándonos en aspectos únicamente formales, la geometría se ha convertido en la herramienta de la que dispone el

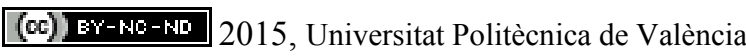


arquitecto para luchar contra el minimalismo impuesto durante las primeras décadas del siglo XX.

"Liberada la arquitectura del recurso del ornamento, la geometría se convierte de esta manera en la responsable de la expresión arquitectónica" (Crespo 2005)

El uso de las superficies geométricas regulares y totalmente definidas ha ayudado a resolver de manera natural e intuitiva problemas constructivos y estructurales a lo largo de la historia de la arquitectura. No es el objeto de la presente comunicación, pero por realizar un acercamiento a este aspecto de la historia de la arquitectura, hay que destacar el hábil uso de la geometría en las bóvedas y cúpulas de la antigüedad que permitió en su día la cubrición de grandes espacios y construir edificios que de no haber sido "geometrizados" no se podrían haber materializado. Ya a finales del s XIX y comienzos del s XX y debido al desarrollo de nuevos materiales como el acero aplicado en edificación o el hormigón armado con su moldeablidad, el repertorio formal de la arquitectura se enriqueció. Por destacar un material, el resultado del uso expresivo del hormigón (Such 2005) dio como resultado un novedoso repertorio formal. Además de repetir formas clásicas como las bóvedas cilíndricas o las cúpulas esféricas, aparecieron nuevas superficies como las superficies regladas de doble curvatura: Paraboloide hiperbólico, hiperboloide hiperbólico, conoide... posteriormente veremos que también se han utilizado superficies irregulares gracias a la moldeabilidad del material.

Con la aparición de las curvas y superficies de Bezier a finales de los años 60 provenientes de la industria automovilística, aplicadas posteriormente a los programas de dibujo asistido por ordenador, el diseño de una nueva arquitectura que ya no solo se basa en formas regladas sino en todo tipo de formas orgánicas e irregulares se hizo posible. Las consecuencias del uso de estos programas y "superficies informatizadas" han llevado a una gran heterodoxia arquitectónica, llena de formas caprichosas a modo de esculturas gigantescas con una amplia libertad de concepción pero con una total indiferencia a las leyes de la física.

"Extraño manierismo adolescente con el imperdonable pecado de la aconstructividad" (Araujo 2005)

Se hace por tanto necesaria una formación de nuestros estudiantes en los principios básicos de la geometría aplicada a la arquitectura, y en concreto, en el conocimiento de las superficies geométricas de mayor uso en la edificación y que gracias a sus singulares características resuelven los problemas constructivos y estructurales de una manera óptima y eficaz. 
Refuerzo de Competencias interdisciplinares en las titulaciones de Arquitectura e Ingeniería de Edificación. Workshop docente "Estructuras singulares"

\section{Uso de las maquetas como herramienta en arquitectura}

La maqueta como herramienta de diseño y experimentación en arquitectura se ha utilizado desde muy antiguo. Los ensayos sobre modelo físico reducido ya los utilizó Leonardo Da Vinci en el renacimiento y más recientemente Antoni Gaudí, siempre en búsqueda de la geometría más eficiente para construir formas novedosas eficientes. Arquitectos como Félix Candela, Frei Otto, Pier Luigi Nervi... e ingenieros como Eduardo Torroja 1960, Heinz Isler, Heinz Hossdorf... comenzaron a realizar ensayos "científicos" sobre modelos físicos reducidos, que aunque con un carácter diferente, buscaban cuantificar el uso de las maquetas en búsqueda de la geometría más eficaz desde el punto de vista estructural(Cassinello 2006).

Podríamos decir que las maquetas han ayudado en la historia de la arquitectura a buscar geometrías y formas novedosas y comprender su funcionamiento estructural, así como las propiedades intrínsecas de los materiales utilizados para construirlas. Esto es lo que ha llevado a desarrollar metodologías basadas en el ensayo experimental sobre modelos físicos reducidos (Andrade 1999).

Desde el punto de vista pedagógico, el modelo físico construido por el alumno ayuda a una mejor comprensión de los principios de una forma, sus características geométricas, sus leyes de generación... aspectos que resultan mucho más difíciles de transmitir con los medios gráficos y los sistemas de representación tradicionales (Sanchis 2013).

\section{El contexto - EXCO, salón internacional de la construcción}

Como ya viene siendo tradicional, desde la ETS de Ingeniería de Edificación se ha organizado en Feria Valencia la $29^{\text {a }}$ edición de EXCO, Salón Internacional de la Construcción, con motivo de CEVISAMA 2015, que tuvo lugar los días 11, 12 y 13 de febrero.

Durante esos días, y en horario de mañana y tarde, se organizaron una serie de conferencias sobre temas relacionados con la edificación, en las que participaron profesores de diversas universidades y técnicos con amplia experiencia profesional. También se realizó una exposición de "Tecnología e Investigación Científica en Edificación” en la que participaron profesores de la ETS de Ingeniería de Edificación.

De forma paralela a estas actividades y como novedad, en esta edición se organizaron una serie de "workshops" sobre temas relacionados con la construcción, que tienen como objetivo la participación de los alumnos y pretenden ser un complemento a su formación técnica. En este contexto y con metodologías de aprendizaje basado en proyectos (ABP) por su dinamismo, teoría y sus diferencias con metodologías tradicionales Kubiatki (2011) llevamos a la práctica el taller de trabajo que se refleja en esta comunicación y que a continuación se describirá con detalle. 


\section{Objetivos}

Los objetivos perseguidos por este workshop; dirigido a alumnos pertenecientes a diferentes grados y niveles, eran fundamentalmente tres:

- Comprobar o reforzar la competencia interdisciplinar "Diseño de superficies singulares plegables"

- Fomentar el trabajo en equipo (primero en equipos pequeños, compitiendo entre ellos, más tarde en un único equipo común)

- Reforzar la motivación intrínseca del alumnado hacia disciplinas académicas separadas de la ejecución.

- Aplicar la resolución fragmentada o modular de problemas a una estructura espacial (de un problema complejo se estudia una célula base primero, y se comprenden las limitaciones en pequeña escala, de manera que al aplicarla a todo el conjunto hay muchos problemas superados)

\section{Desarrollo de la innovación}

Como ya se ha comentado en la introducción, en el ámbito de la Feria de la Tecnología de la Construcción EXCO 2015, y como viene ocurriendo desde hace años y es tradición en la Escuela Técnica Superior de Ingeniería de Edificación (ETSIE), toda la docencia se traslada durante su duración a las aulas de Feria Valencia. En esta edición desde la dirección de EXCO siguiendo con la iniciativa experimentada el año anterior y en el ámbito de apostar por nuevas metodologías docentes activas y enfocadas al espíritu del nuevo Espacio Europeo de Educación Superior, se ofertaron talleres formativos para los alumnos.

La ETSIE puso a disposición de los docentes el entorno y, hasta donde fuera posible, el material para que aquellos docentes invitados pudiesen organizar cualquier tipo de taller que se saliese de la docencia reglada y estuviese enfocado a las competencias profesionales de la titulación. La normativa marcada por parte de la organización de EXCO consistía en la realización de los talleres el día 11 de febrero de 15 a 18h. Esto nos daba una duración de 3h para la realización total del taller. El resultado del mismo se podría exponer durante los días 12 y 13 en el Centro de Eventos de Feria Valencia. Para todo ello la dirección nos proporcionó áreas de trabajo acordes a las necesidades del taller, suministro eléctrico, limpieza y una subvención para los materiales empleados en el mismo. En este contexto por parte de la dirección de la organización del evento se invitó a 3 equipos de docentes la realización de 3 talleres distintos. Se ofertó un taller de bóvedas funiculares, otro de aprender construyendo y el descrito en la presente comunicación, la invitación se realizó en base a los resultados de los talleres ofertados la anterior edición.

En este entorno los autores plantearon la posibilidad de realizar un taller en el que aunar competencias del área de conocimiento de las asignaturas de proyectos, estructuras,

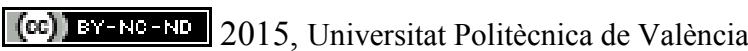


Refuerzo de Competencias interdisciplinares en las titulaciones de Arquitectura e Ingeniería de Edificación. Workshop docente "Estructuras singulares"

construcción y ejecución. En base a nuestras experiencias docentes e investigadoras decidimos aunar conocimientos de todos los autores y aplicar la metodología activa de aprendizaje basado en proyectos (ABP) para la ejecución del taller de estructuras singulares.

Con los objetivos establecidos y la aceptación del planteamiento por parte de la dirección de EXCO, los primeros trabajos consistieron en el diseño detallado de la actividad decidiendo el tipo de estructura que queríamos ejecutar. En base a curiosidades personales y como homenaje al arquitecto Emilio Pérez Piñeiro decidimos ejecutar una estructura plegable realizada con materiales básicos Figura 1.

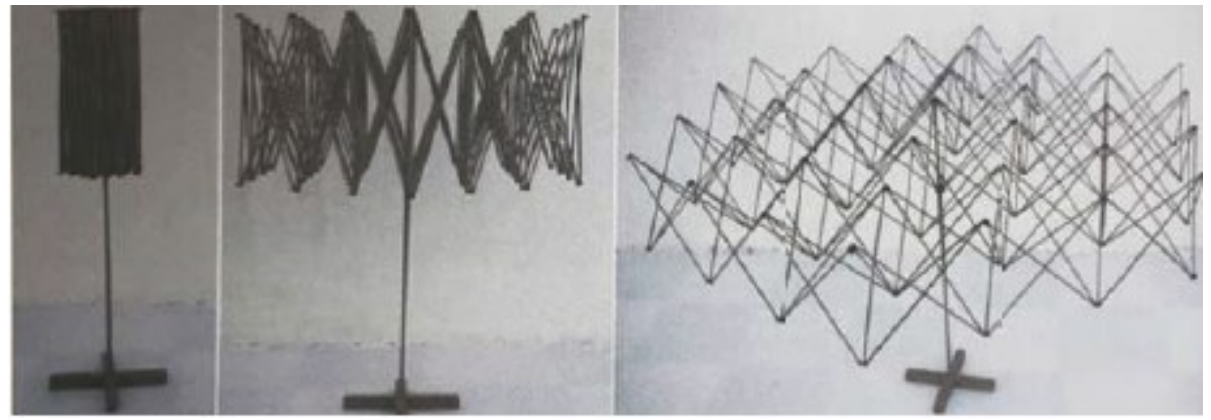

Figura $1 \cdot$ Estructura plegable de módulo cuadrado Escrig (2012)

Dado el carácter del taller y su duración la simplificación de la misma era obligada con lo que optamos por las formas más sencillas que el arquitecto de corto recorrido pero amplio reconocimiento internacional había estudiado a mediados del siglo pasado y que el profesor José Félix Escrig Pallarés Doctor en Arquitectura de la Escuela de Sevilla había desarrollado más en profundidad. Siguiendo la publicación de Escrig (2012) optamos por el desarrollo de uno de los módulos más sencillos de realizar el de base cuadrada Figura 2.

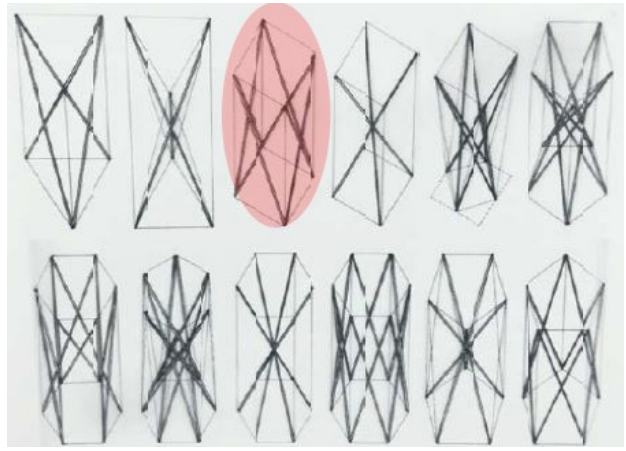

Figura 2· Módulos base patentados por Félix Escrig, Escrig (2012) 
Una vez decidido el módulo establecimos los parámetros que queríamos alcanzar con el proyecto que íbamos a lanzar a los alumnos. La elección de materiales reciclados o básicos era una condición intrínseca del taller con tal de acercar la edificación a la sencillez y facilidad de ejecución (Figura 3). Los materiales que necesitábamos eran elementos que materializaran las barras y los nudos, así como un elemento tirante o rigidizador. Para todos los elementos se barajaron distintas opciones que se ensayaron para la toma de decisión.

- Las barras se plantearon como listones de madera, barras de metal optándose finalmente por palos de escoba, que le conferían la rigidez suficiente, fácil manipulación y era un elemento muy accesible.

- Los nudos y su geometría se trataba del elemento más complejo y donde reside la magia de las estructuras plegables. Se barajaron todo tipo de elementos prefabricados, se visitaron numerosas ferreterías. Se diseñó un nudo que se imprimió con tecnología FDM con una impresora 3D con un resultado óptimo pero alto precio. Al final se optó por el uso de escuadras de $90^{\circ}$ zincadas con doble hueco centradas y su unión con tornillería de métrica 4.

- El atirantamiento se dejó como decisión final de los alumnos teniendo posibilidades de ejecutarlo con barras restantes o mediante un cableado de las diagonales.

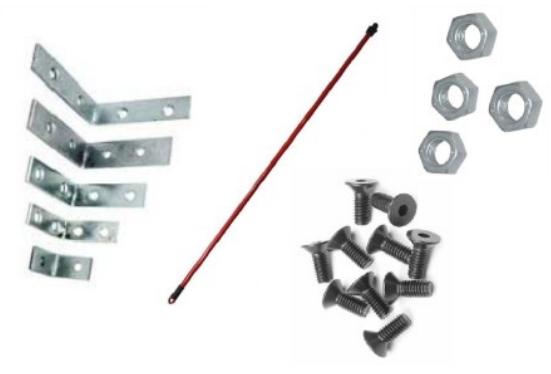

Figura $3 \cdot$ Materiales utilizados

Una vez seleccionados todos los elementos que serían utilizados en el desarrollo del taller se hacía necesaria la realización de prototipos funcionales (Figura 4). Los prototipos debían conseguir una doble función, por un lado comprobar que los elementos seleccionados permitirían la materialización de la estructura y además comprobar el funcionamiento del mecanismo de plegado con el nuevo nudo creado. Se realizaron 2 tipos de prototipos, uno esquemático con brochetas y gomas para confirmar las hipótesis de montaje y plegado y uno segundo más elaborado con la elección de los materiales y sobretodo la ejecución de los nudos inventados para este taller. 
Refuerzo de Competencias interdisciplinares en las titulaciones de Arquitectura e Ingeniería de Edificación. Workshop docente "Estructuras singulares"

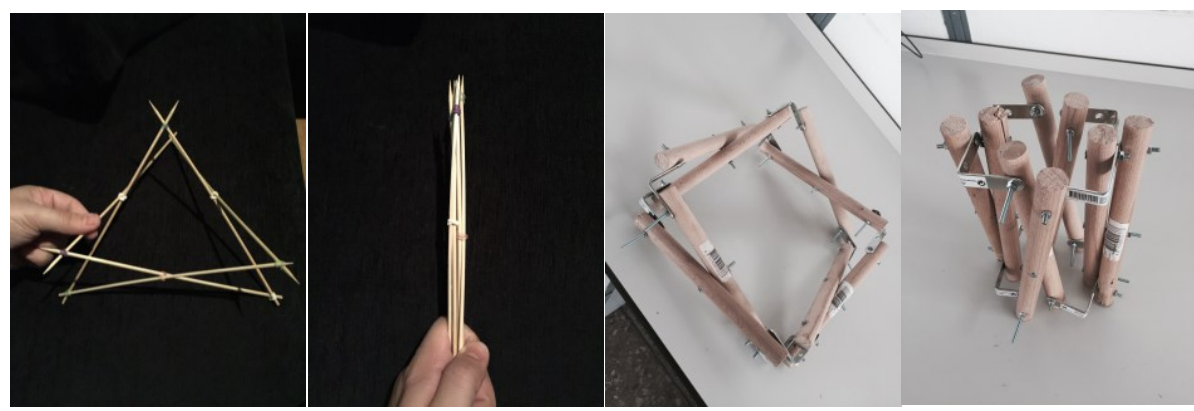

Figura $4 \cdot$ Prototipos iniciales

Con tal de alcanzar los objetivos planteados con el módulo fijado, los materiales elegidos y el prototipo funcionando, se realizó un anteproyecto de la estructura que se propondría ejecutar a los alumnos. El proyecto para los alumnos consistiría en la realización de una estructura plegable realizada con materiales básicos que cubriese una superficie aproximada de $3 \times 3 \mathrm{~m}$. Para ello a los alumnos se les facilitó el proyecto, el material a utilizar y las herramientas necesarias para su ensamblaje.
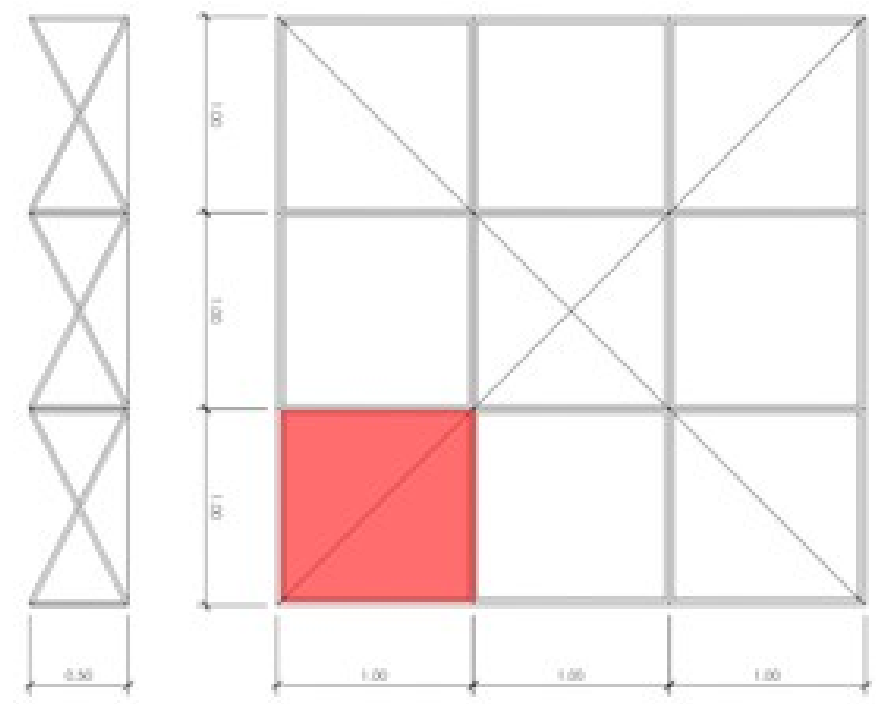

Figura $5 \cdot$ Proyecto de estructura plegable planteada a los alumnos y modulo tipo

Dada la difusión realizada por la EXCO, al taller se inscribieron el número máximo de alumnos permitidos, establecido en 10, 7 de la ETSIE y 3 de la Escuela Técnica Superior de Arquitectura (ETSA) ambas de la Universitat Politécnica de Valéncia (UPV). El número de alumnos viene determinado por el número de tareas a acometer durante el proceso de ejecución y el número de equipos. Se plantearon 5 equipos de 2 personas los cuales 
ejecutarían 2 módulos (Figura 5) cada uno quedando el modulo central más complejo para el ultimo equipo.

Las 3 horas de duración del taller se estructuraron en 4 fases claramente diferenciadas:

- 30 minutos de explicación histórico-teórica.

- 60 minutos para la realización de los módulos asignados a cada uno de los equipos pequeños.

- 75 minutos de ensamblaje del todo en el gran grupo.

- 15 minutos de atirantado y montaje exposición.

La primera fase consistió en la explicación histórica de las estructuras plegadas dejando claros los conceptos de sus preceptores y creadores así como de la bibliografía básica existente al respecto. A cada alumno se le entregó un dossier de 7 folios con la explicación de todo lo necesario para el desarrollo teórico-práctico del taller. En esta fase se explicó también el proyecto a desarrollar, los materiales seleccionados y su porqué así como las herramientas de las que disponían para su realización que eran taladro, caladora y herramienta habitual de mano. Una parte importante fue establecer un vocabulario común para poder entender los distintos elementos que formarían la estructura como eran los módulos y las tijeras, de esta manera era más fácil organizar las tareas y posteriormente el montaje final de ensamblado.

\section{La segunda fase (}

Figura 6) de 1 hora de duración consistió en formar 5 equipos de 2 personas. Cada uno de estos pequeños equipos se formó de manera libre y a cada equipo se les asignó la ejecución 2 módulos (Figura 5) del todo estructural. Los alumnos debían en esta fase cortar las barras a la medida establecida, perforar los agujeros necesarios para las uniones entre barras y los nudos y montar cada uno de los 2 módulos de manera independiente. Finalizaban la fase con los 2 módulos completados y funcionando, es decir plegándose y desplegándose igual
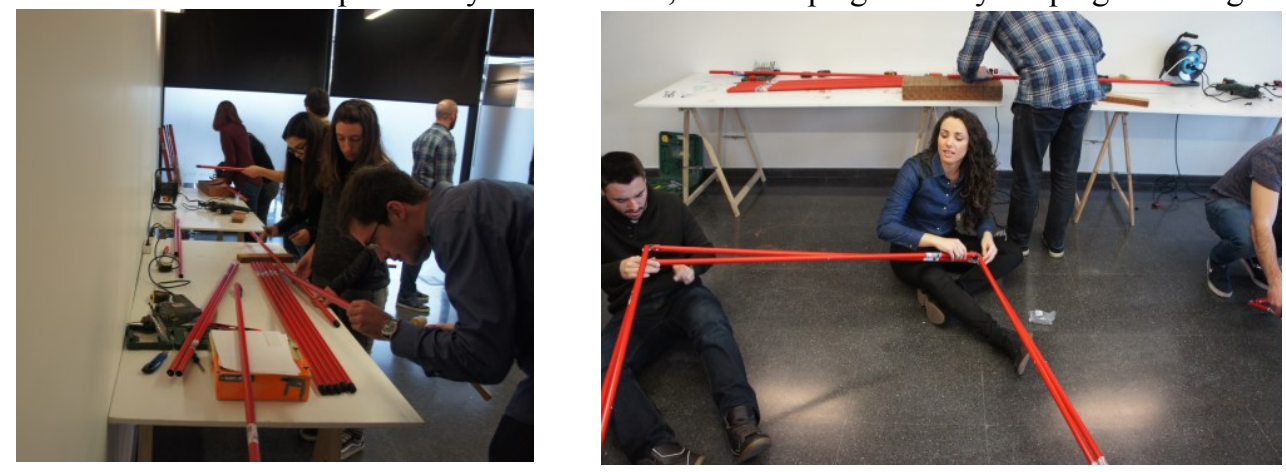

que los prototipos descritos anteriormente (Figura 4).

\section{(cc)) EY-NC-ND 2015, Universitat Politècnica de València}


Refuerzo de Competencias interdisciplinares en las titulaciones de Arquitectura e Ingeniería de Edificación. Workshop docente "Estructuras singulares"

Figura $6 \cdot$ Fase 2 de montaje

La tercera fase (Figura 7) de hora y media ya se realiza en el gran grupo. Cada pequeño grupo debe aportar su parte de trabajo y ponerse en común con los criterios de montaje con el gran grupo para ser capaces de ensamblar los distintos módulos entre sí. Esta fase resultó especialmente complicada dada que la posición de las escuadras respecto a las barras y entre si no podía ser arbitraria sino que tenía que seguir un orden y criterio absolutamente estricto y en concordancia con su posición en el todo. Es en este punto donde el liderazgo, la capacidad espacial de iniciativa y resolución salen a relucir. Virtudes estas muy necesarias en el sector de la edificación, resultando este un ejercicio muy válido para valorar las capacidades de los alumnos.
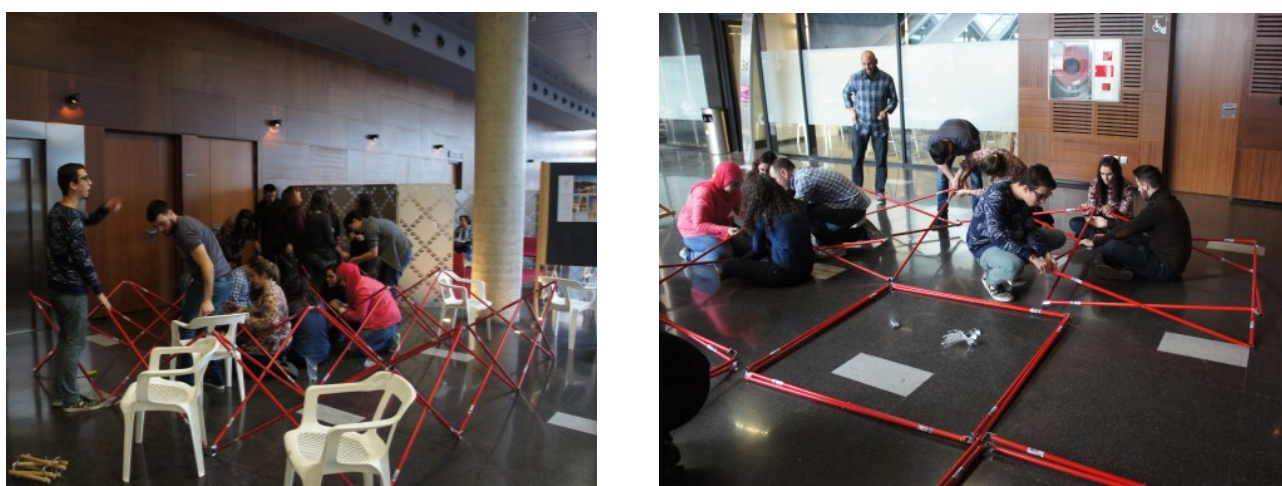

Figura $7 \cdot$ Fase 3 de ensamblaje

Por ultimo en la fase 4, durante los últimos 15 minutos y una vez las 3 fases previas estaban completadas se finalizó el taller realizando el atirantamiento de la estructura y montando la exposición del mecanismo ejecutada. Para ello se atirantó la misma para eliminar un grado de libertad y acotar su movimiento en el eje z, decisión que viene determinada por la inercia necesaria para la estructura, es decir por su canto, conceptos todos ellos trabajados durante su ejecución. En este punto se dio libertad a los alumnos para hallar la solución óptima con el material disponible y por consenso se llegó a la solución de atirantar las diagonales mayores de la estructura mediante una lienza (Figura 5). Una vez atirantada la estructura se preparó la exposición elevando la misma sobre 4 caballetes para dotar de altura y entidad a la misma. También se acompañó la estructura de una sencilla explicación del taller así como de los prototipos realizados en la fase de preparación del taller (Figura 8). 

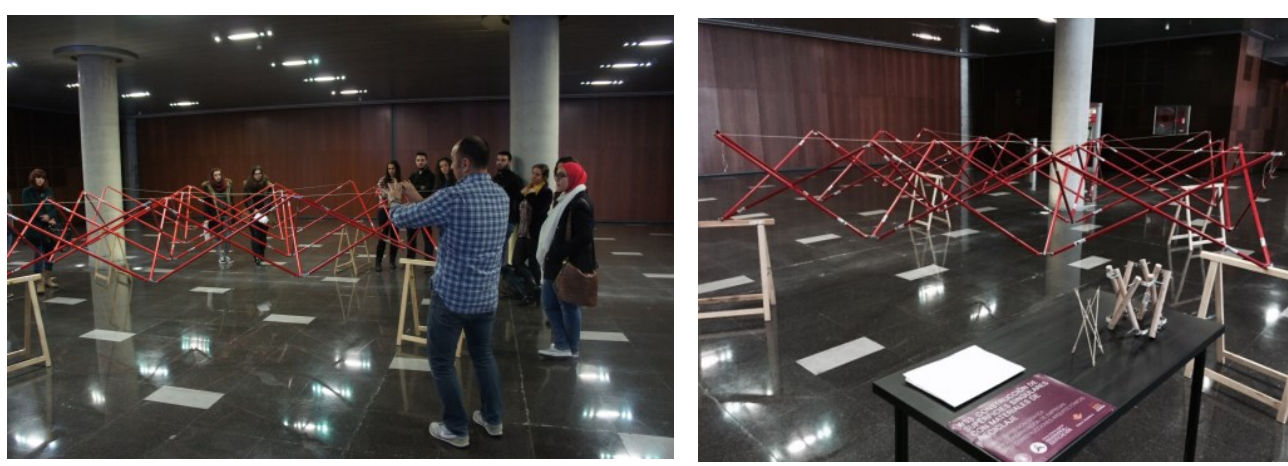

Figura $8 \cdot$ Atirantado y exposición final

\section{Resultados}

Los resultados se pueden analizar desde 2 puntos de vista, el cualitativo y el cuantitativo.

A nivel cuantitativo en los días posteriores a la realización del taller se les pasó una encuesta on-line a los participantes con el uso de herramientas de trabajo colaborativo. La encuesta constaba de una parte objetiva descriptiva de la muestra con 5 preguntas, y una parte subjetiva de valoración del taller con otras 5 preguntas con una escala tipo Likert de 5 niveles, más una última pregunta final de valoración global del taller con una escala de 1 a 10. La encuesta se les envió a los 10 integrantes del taller y respondieron 8 alumnos, resultando el $80 \%$ de la población muestral.

\begin{tabular}{|cc|ccccccc|cc|cc|}
\hline \multicolumn{2}{|c|}{ Género } & \multicolumn{5}{|c|}{ Edad } & \multicolumn{2}{c|}{ Escuela } & \multicolumn{2}{c|}{$\begin{array}{c}\text { Participación } \\
\text { anterior }\end{array}$} \\
\hline Hombre & Mujer & 19 & 22 & 23 & 24 & 25 & 28 & +30 & ETSIE & ETSA & SI & NO \\
\hline $62.5 \%$ & 37.5 & 12.5 & 25 & 12.5 & 12.5 & 12.5 & 12.5 & 12.5 & 75 & 25 & 37.5 & 62.5 \\
\hline
\end{tabular}

Como se aprecia en la Tabla 1, la muestra estaba compuesta por 5 alumnos masculinos que representan el $62.5 \%$ frente a un $37.5 \%$ que representan las 3 mujeres participantes. La edad mínima fue de 19 años siendo la máxima de más de 30 existiendo una franja del $62.5 \%$ de la población entre los 22 y los 25 años. El $75 \%$ de los que respondieron pertenecen a la ETSIE y el 25\% restante pertenece a la ETSA. En cuanto al curso predominante al cual pertenecían los alumnos hay disparidad como se puede observar en Figura 9. 
Refuerzo de Competencias interdisciplinares en las titulaciones de Arquitectura e Ingeniería de Edificación. Workshop docente "Estructuras singulares"
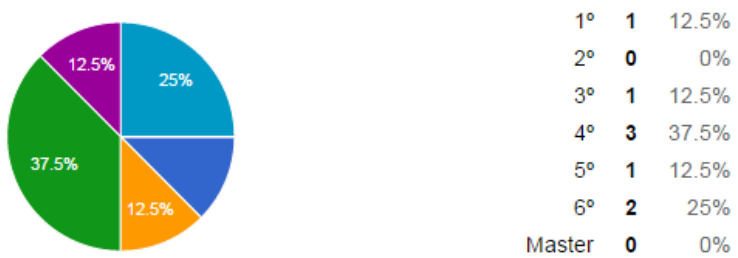

Figura $9 \cdot$ Curso predominante de los encuestados

En cuanto a la evaluación subjetiva del taller por parte de los alumnos los resultados obtenidos son los siguientes:
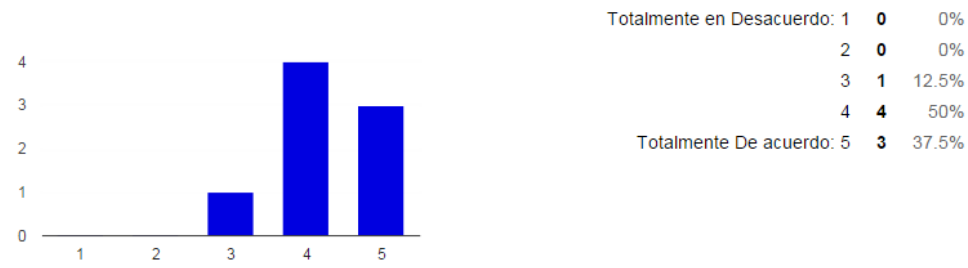

Totalmente De acuerdo: $5 \quad \mathbf{3} \quad 37.5 \%$

Figura $10 \cdot ¿$ ¿La información escrita facilitada ha sido suficiente?

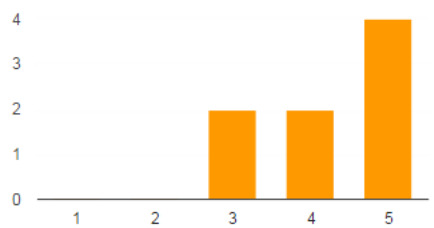

Totalmente en Desacuerdo: $1 \quad 0 \quad 0 \%$

Totalmente De acuerdo: $5 \quad 4 \quad 50 \%$

Figura $11 \cdot$ ¿El material empleado en el taller me ha parecido adecuado?
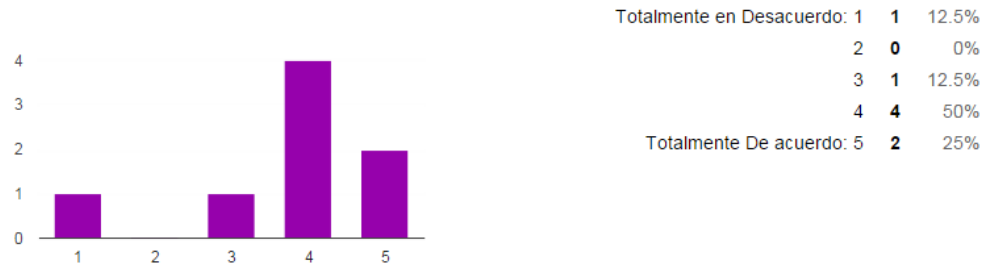

Figura $12 \cdot$ ¿Mejora el taller mis competencias profesionales? 

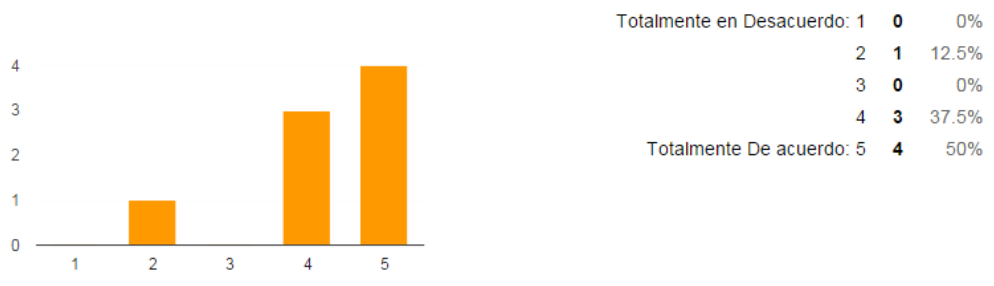

Figura $13 \cdot$ ¿Me parece una metodología docente válida para mi formación?
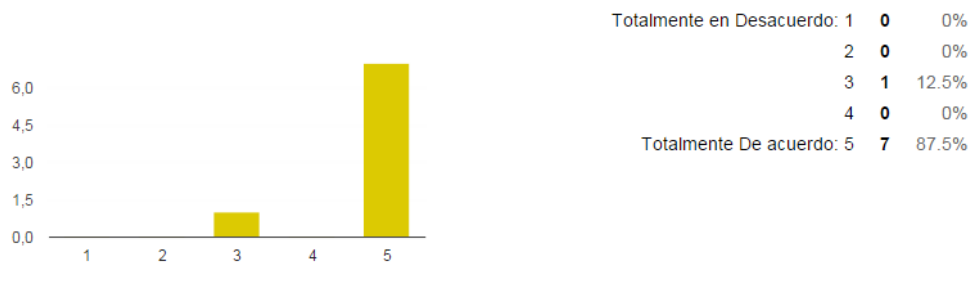

Figura $14 \cdot ¿$ ¿Repetiría una experiencia docente similar?
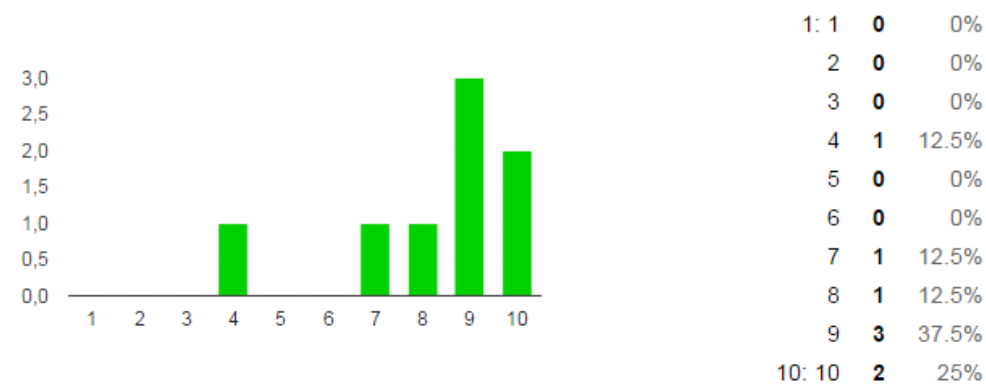

Figura $15 \cdot$ En general mi valoración del taller es...

Los datos arrojan unos resultados positivos a todos los niveles, tanto los tangibles en cuanto a información proporcionada y los materiales empleados, como los asociados a competencias y metodología. Llama la atención que existe una persona que opina que el taller no mejora sus competencias profesionales, posiblemente sea la misma persona que opina que no le parece una metodología docente válida, sin embargo a la pregunta de si repetiría parece obvio que la opinión se desplaza a una respuesta neutral. En todo caso las respuestas positivas (de acuerdo, o totalmente de acuerdo) en todos los conceptos evaluados están por encima del $80 \%$. Y la valoración global del taller es sobresaliente para el $62.5 \%$ de los alumnos.

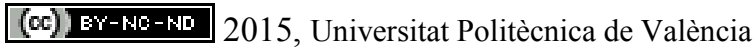


Refuerzo de Competencias interdisciplinares en las titulaciones de Arquitectura e Ingeniería de Edificación. Workshop docente "Estructuras singulares"

Desde el punto de vista cualitativo el taller ha sido tremendamente satisfactorio y se han alcanzado todos los objetivos planteados inicialmente en el mismo. Tanto las inscripciones al taller, como su desarrollo y la motivación intrínseca mostrada por los participantes demuestran sin duda que estas metodologías docentes suponen una propuesta de valor muy interesante para el alumnado. El último ejercicio realizado fue una discusión entre todos los integrantes, docentes incluidos sobre el desarrollo del mismo, tanto en aspectos formales como de contenidos y resultados. En esta discusión se debatieron, procesos, métodos, herramientas, materiales y aspectos que mejorarían el elemento construido. Prácticamente todos los alumnos aportaron algo a la discusión, todos ellos consideraban la actividad como muy enriquecedora y repetirían la experiencia sin dudarlo aplicándolo a otros elementos constructivos. Fue significativo que casi todos ellos tenían aportaciones a las herramientas y materiales empleados dado que esta es una competencia profesional básica del grado.

\section{Conclusiones}

Las conclusiones del proceso docente de refuerzo de competencias arrojan lo que pedagógicamente está sobradamente demostrado, las metodologías activas donde la práctica profesional, real acerca la teoría con la práctica a través de retos motivadores son la chispa necesaria para encender el fuego del aprendizaje.

Tanto los datos cuantitativos como los cualitativos en las distintas experiencias que hemos realizado al respecto los últimos años, nos demuestran que los alumnos activan su motivación intrínseca (lo que lleva a un aprendizaje profundo) cuando realmente son capaces de intuir la conexión entre asignaturas y estas con su próxima realidad profesional.

A modo resumido hemos extraído las siguientes conclusiones:

- Los alumnos han conseguido conectar conceptos que tenían asimilados como independientes. Se ha conseguido ver y enfocar un problema desde diferentes puntos de vista (geometría, arquitectura, estructuras y ejecución) cumpliendo de este modo con nuestro primer objetivo.

- Se ha conseguido trasladar un problema del papel a la realidad.

- Se ha conseguido crear grupos multidisciplinares (estudiantes de arquitectura e Ingeniería de Edificación juntos en los diferentes grupos), lo cual ha sido muy positivo a la hora de reforzar mutuamente los diferentes resultados de aprendizaje. Trabajando de este modo el segundo objetivo planteado en el taller.

- Con los elementos tensionados se ha comprendido perfectamente cómo funciona un tensor (ya que al variar la tensión de los diferentes cables, la estructura variaba de forma)

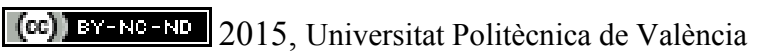


- Sobre una estructura simple (cobertura plana) se han podido plantear elementos más complejos como cúpulas, bóvedas, etc... Trabajando de este modo el cuarto objetivo planteado con la experiencia.

\section{Referencias}

ANDRADE, C. et al. (1999) "Centenario de Eduardo Torroja (Ciencia, Tecnología y Empresa)" en Informes de la Construcción, vol. 51, n 462, p. 5-8

ARAUJO ARMERO, R. (2005) “Geometría, técnica y arquitectura” en Revista Tectónica 2005 n 17 Madrid.

CASSINELLO, P. (2006) “En Memoria de Heinz Hossdorf” en Informes de la Construcción, vol. 58, $\mathrm{n}^{\mathrm{O}} 502$.

CRESPO CABILLO, I. (2005) Control gráfico de formas y superficies de transición. Tesis doctoral. Barcelona: Universitat Politècnica de Catalunya

ESCRIG, F., ESCRIG PALlARÉS, F., PÉREZ VALCARCEL, J. (2012) Modular, ligero, transformable: Un paseo por la arquitectura ligera móvil Sevilla: Universidad de Sevilla.

JORDÁ SUCH, C. (2005) "El hormigón armado y el desarrollo de la tipología laminar: la transformación del canon en la arquitectura moderna" IV Congreso Nacional de Historia de la Construcción. Cádiz.

SANCHIS SAMPEDRO, F J. (2013) "Las maquetas como herramienta docente en la enseñanza de la Geometría Descriptiva". $35^{\circ}$ Convegno internazionale dei docenti della rappresentazione. Matera, Italia.

TORROJA, E. (1960) Razón y ser de los tipos estructurales. Madrid: CSIC. 